\title{
Fast Timing Detectors towards a 4-Dimensional Tracking
}

${ }^{a, b}$ V. Sola*, ${ }^{b, c}$ R. Arcidiacono, ${ }^{b}$ N. Cartiglia, ${ }^{a, b} \mathbf{M}$. Ferrero, ${ }^{b} \mathbf{M}$. Mandurrino, ${ }^{b} \mathbf{A}$. Staiano

a: Università di Torino, Torino, Italy

${ }^{b}$ : INFN, Torino, Italy

${ }^{c}$ : Università Piemonte Orientale, Novara, Italy

E-mail: valentina.solaeto.infn.it

In this contribution we review the growing interest in implementing large-area fast-timing detectors with a time resolution of $30-35$ ps, based on Low-Gain Avalanche Detectors. Precise time information added to tracking brings benefits to the performance of the detectors by reducing the background and sharpening the resolution; it improves tracking performances and simplifies tracking combinatorics. Large-scale high-precision timing detectors have to face formidable challenges in almost every aspect: sensors performance, segmentation and radiation tolerance, very low-power and low-noise electronics, cooling, low material budget, and large data volumes. We will report on the current status and new development of such detectors for high energy physics, in view of their possible use in the experiment upgrades at the High Luminosity LHC and beyond.

The 39th International Conference on High Energy Physics (ICHEP2018)

4-11 July, 2018

Seoul, Korea

${ }^{*}$ Speaker. 


\section{Introduction}

The new era of particle accelerators aim to increase at the same time instantaneous luminosity and centre-of-mass energy, resulting in a very high density of particles generated at each bunch crossing. In such environment, precise timing information together with tracking information will be crucial to disentagle different events, helping in rejecting the backgrounds. This is the basic concept of the so-called 4-dimensional (4D) tracking.

Timing detectors are extremely specialised sensors, and each operating condition and geometry requires a different choice of optimum technology. Depending on how detectors are build, timing information can be available at different stages in the reconstruction of an event. The best option is when timing is associated to each point of the track. Candidates for such technological challenge are the Ultra-Fast Silicon Detectors (UFSD) [1], innovative silicon sensors optimised for timing measurements based on the Low-Gain Avalanche Diode (LGAD) design.

UFSD sensors with appropriate electronics are able to provide accurately space-time coordinates, however particle rate add a further constraint to the design of the detcetor. Particle rate is a key factor in the overall detector as it impacts the front-end real estate, the power consumption and the data transfer rate. To achieve a 4D tracking system in a high-rate high-density environment all the above aspects have to be extremely performing (see Fig. 1).

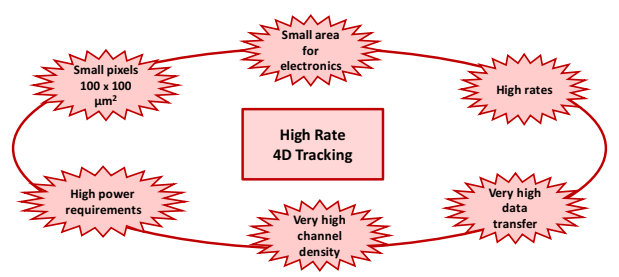

Figure 1: Interconnection of several aspects of space-time tracking at high rate.

\section{Ingredients of the Time Resolution}

The time resolution of a detector, $\sigma_{t}$, results from the sum of different contributions, namely (i) the jitter, $\sigma_{\text {Jitter }}$, (ii) the Landau Time Walk, $\sigma_{T W}$, the Landau noise, $\sigma_{\text {Landau Noise }}$, (iv) the signal distortion, $\sigma_{\text {Distortion }}$, and (v) the TDC binning, $\sigma_{T D C}$ :

$$
\sigma_{t}^{2}=\sigma_{\text {Jitter }}^{2}+\sigma_{T W}^{2}+\sigma_{\text {Landau Noise }}^{2}+\sigma_{\text {Distortion }}^{2}+\sigma_{T D C}^{2} .
$$

For the results presented here, the following simplification can be applied:

i The effect of time walk can be compensated using Constant Fraction Discriminator (CFD) or Time-over-Threshold (ToT) analyses;

ii The $\sigma_{\text {Distortion }}$ term is driven by two components: non-uniform weighting field, and nonsaturated drift velocity. Both terms are made small by using a parallel plate geometry and operating the sensor at a bias voltage at which the carriers' velocities are saturated.

iii The TDC binning can be chosen properly, to keep $\sigma_{T D C}$ contribution below $10 \mathrm{ps}$. 
Assuming these simplifications, the predominant terms to the time resolutions are jitter and Landau noise.

\section{Sensors for $4 D$ Tracking}

Ultra-Fast Silicon Detectors (UFSD) are innovative silicon sensors optimised for timing measurements based on the Low-Gain Avalanche Diode (LGAD) design. LGAD merges the best characteristics of traditional silicon sensors with the main feature of Avalanche Photodiodes (APD), using n-in-p silicon diodes with a low and controlled internal multiplication mechanism. To obtain charge multiplication, an electric field of the order of $\mathrm{E} \sim 300 \mathrm{kV} / \mathrm{cm}$ is required, under this condition the electrons (and to less extent the holes) acquire enough kinetic energy that are able to generate additional e/h pairs. The required field value is obtained by implanting an appropriate acceptor density under the n-p junction.

With respect to standard silicon sensors, UFSD have the advantage of having a large signal, with a fast and steep rise, and therefore are able to minimise the jitter. The Landau contribution, instead, depends strongly on the sensor thickness, and moderately on the gain value.

UFSD are routinely produced by three vendors (CNM, FBK, and HPK) and have demonstrated that $50 \mu$ m-thick sensors reach a time resolution of $30-35$ ps with a signal multiplication of about 20 - 25. Figure 2 shows the UFSD time resolution as a function of the sensor thickness, and display the separate contribution of Landau and jitter terms, as simulated by Weightfield2 (WF2).

At present the UFSD technology allow to reach a time resolution of $\sim 35 \mathrm{ps}$ up to fluences above $10^{15} 1 \mathrm{MeV} \mathrm{n}_{e q} \cdot \mathrm{cm}^{-2}$ and better of $\sim 60 \mathrm{ps}$ up to fluences above $10^{16} 1 \mathrm{MeV} \mathrm{n}_{e q} \cdot \mathrm{cm}^{-2}$ $[2,3]$, and $R \& D$ is still ongoing to further improve the radiation tolerance.

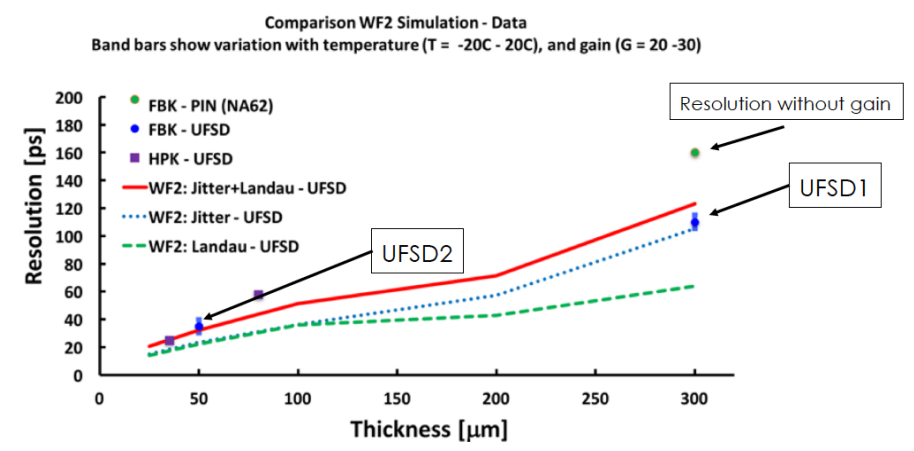

Figure 2: UFSD time resolution as a function of the sensor thikcness. The time resolution of a standard silicon device (green dot) is shown as a reference.

\section{Electronics Challenge for 4D Tracking}

To achieve ultimate 4D tracking capability, the pixel size has to reach dimensions of $\sim 100 \times 100$ $\mu \mathrm{m}^{2}$. However, fitting the necessary electronics in the corresponding area on the read-out chip, in environment with high particle rates, is very challenging. The precise measurement of time needs quite a bit of electronics, and always a TDC. Moreover, at high rate, the TDC digitisation speed might not be enough to keep up with the particle rate, and more than one TDC is needed for each 
pixel. Therefore, the particle rate has a direct impact on the minimum dimension of the pixels[4]. In the $130 \mathrm{~nm}$ technological node a TDC needs approximately $\sim 100 \times 100 \mu \mathrm{m}^{2}$, that scales to $\sim 50 \times 50 \mu \mathrm{m}^{2}$ in the $65 \mathrm{~nm}$ node, and hopefully, to $\sim 25 \times 25 \mu \mathrm{m}^{2}$ in the $28 \mathrm{~nm}$ one. Hence, high rate and small pixel size require the use of the smallest possible technological node available. The situation is further complicated by the large amount of data volume generated by several TDCs working in parallel in each pixel, and by the need to remove the power used by the analog front-end and digital electronics.

Of equal importance, but not discussed in this article, are the new algorithms and read-out architectures, as those presented in [5], needed to fully exploit the 4D tracking at high rates.

\section{Outlook}

The concurrent measurement of space and time in high energy physics experiments is attracting a lot of attention due to the need to overcome very high densities and high energies, starting from the High Luminosity LHC (HL-LHC). Following the recent development in silicon detectors, UFSD technology have demonstrated the capability to achieve a precision of $30-35 \mathrm{ps}$, and to retain good performances up to fluences of $5 \cdot 10^{15} 1 \mathrm{MeV} \mathrm{n}_{e q} \cdot \mathrm{cm}^{-2}$ [3].

UFSD are the detector chosen by the ATLASand CMSCollaborations for the HL-LHC upgrade, to provide a precise timing measurement to each charged particle that cross the tracking detector volume. To this end, several production of UFSD are planned by three foundries (CNM, FBK, and HPK) in the next couple of years, with the goal of testing and improving (i) radiation tolerance, (ii) gain uniformity, (iii) sensor size, and (iv) fill factor.

\section{Acknowledgments}

We acknowledge the fundamental contributions coming from the discussions, and active collaboration of the RD50 colleagues. Part of this work has been financed by the European Union's Horizon 2020 Research and Innovation funding program, under Grant Agreement no. 654168 (AIDA-2020) and Grant Agreement no. 669529 (ERC UFSD669529), and by the Italian Ministero degli Affari Esteri and INFN Gruppo V.

\section{References}

[1] H. Sadrozinski, A. Seiden, N. Cartiglia, 4-Dimensional Tracking with Ultra-Fast Silicon Detectors, Rep Prog Phys. 81 (2018) 026101. doi:10.1088/1361-6633/aa94d3

[2] M. Ferrero et al., Radiation resistant LGAD design, submitted to Nucl.Instrum.Meth. A. arXiv:1802.01745

[3] S.M. Mazza et al., Properties of HPK UFSD after neutron and proton irradiation up to $6 \cdot 10^{15}$ $n_{e q} / \mathrm{cm}^{2}$. arXiv:1804.05449

[4] S. Cadeddu et al., A time-to-digital converter based on a digitally controlled oscllator, at 2016 IEEE-NPSS Real Time Conference (RTC 2016). doi:10.1109/RTC.2016.7543099

[5] N. Neri et al., 4D fast tracking for experiments at high luminosity LHC, J.Inst. 11 (2016) C11040. doi:10.1088/1748-0221/11/11/C11040 